\title{
Study on Modeling Method of the Precision Machined Surface Geometry form Error Based on Bi-Cubic B-Spline
}

\author{
Z.Q. Zhang, X. Jin \\ School of Mechanical Engineering \\ Beijing Institute of Technology \\ Beijing, China
}

\author{
X. Jin \\ School of Mechanical Engineering \\ Beijing Institute of Technology \\ Beijing, China
}

\author{
Z.J. Zhang \\ School of Mechanical Engineering \\ Beijing Institute of Technology \\ Beijing, China
}

\begin{abstract}
For precision mechanical system, the different 3d spatial distribution of form error causes different assembly contact state. Since 3d spatial distribution of form error is not taken account in modeling and evaluation method of geometric form error and it is hard to quantitatively analyze the relationship between form error and assembly accuracy of precision mechanical system, a modeling method of machined surface form error based on bi-cubic B-spline surface reconstruction is proposed. Firstly, a reconstruction algorithm of CMM date based on bi-cubic B-spline is proposed, so the mathematical model of the surface form error is established. Then, the data transaction method between Matlab and Pro/E is proposed, and the 3D solid model of parts is established, which has the surface form error. Finally, the validity and reliability of the proposed method is validated by the establishment of the typical mating surfaces form error model.
\end{abstract}

Keywords-form errors; assembly; bi-cubic b-spline; surface reconstruction

\section{INTRODUCTION}

As everyone knows, the geometrical error of parts surface has been a key factor of assembly performance analysis for precision mechanical system. Because mechanical part surfaces cannot be smooth planes actually, the geometrical error has quite an effect on assembly performance. For precision mechanical system, machining errors include dimension error, geometric form error and position error, usually affect the assembly accuracy fundamentally. High precision assemblies cannot be analyzed with the assumption that form errors are negligible. The dimension error and position error are scalars, which can be relatively simply considered in assembly. However, the influencing mechanism of the form error on the assembly accuracy is more complex due to the complexity of form error distribution. The form error and assembly force result in non-uniform contact state between mating surfaces, and thus non-uniform stress in components, which will degrade the assembly accuracy. In order to make quantitative analysis on the surface geometric error and analyze the effective rules of the geometrical error on assembly accuracy, it is necessary to establish an accurate $3 \mathrm{~d}$ model of the geometrical error.

In the last 30 years, various methods have been developed to characterize the machined surfaces. A high order polynomial function to model form tolerance for nominal planner part feature by Turner [1]. The Fourier series is the first used in the decomposition method in order to define periods on circular by Goto and Iizuka [2].Henke et al. [3] worked on the geometrical variations of cylindrical surfaces and matched the Chebyshev polynomials with Fourier series to evaluate systematic manufacturing defects. Two dimensional Fourier is used by Capello and Semeraro [4] to define the form parameters of rectangular shapes. Serge Samper [5] proposed a new way to define form error parameters based on the eigen-shapes of natural vibrations of surfaces. Huang and Ceglarek [6] used the discrete cosine transform (DCT) to describe rectangular surfaces. Fractal theory and wavelet analysis theory is used by Srinivasan [7] to analyze the form tolerances, and a two-dimensional model of the shape error is established

The above research is limited to decomposition and reconstitution of the machined form error and the errors are too big. Furthermore, it is difficult to establish the accurate 3D model of surface form error. In order to solve these problems, this paper puts forward a new modeling method of the machined surface geometry form error for precision assembly, the three-dimensional distribution of form error can be described more accurately, assembly technology for precision mechanical system can be effectively designed, the assembly precision and precision stability can be improved.

\section{Methodology}

\section{A. Modeling Method of Form Error Based on Bi-Cubic} $B$-Spline Surface Reconstruction

The surface geometric errors of parts are mainly caused by processing technology because of force deformation and 
thermal strain. The surface is consistent with the physical characteristics of shape modification of B-spline Surface. Spatial curved surface is described with B-spline Surface, which has been widely applied in practical engineering. Because of the local support property and $C^{2}$ continuous of B-spline Surface, it has been widely applied in CAD/CAE. Surface Reconstruction Based on bi-cubic B-spline surface of Points Cloud Data from CMM is proposed in this paper and solid model of the part is established using the three-dimension CAD software Pro/E. Firstly an algorithm for inverse calculation of cubic B-spline is introduced, and based on this evidence, this paper proposes an algorithm based on bi-cubic B-spline surface interpolation.

\section{B. Reverse Calculating the Control Points of Cubic B-Spline}

In order to make cubic B-spline curve through a set of data points $\left(P_{i}, i=0,1, \cdots, n\right)$, the endpoint of B spline curve is made respectively with the first and last data points consistent in the process of inverse calculation. Interpolation equation of cubic B-spline is represented as Eq. (1).

$$
P_{i}(u)=\sum_{j=i-3}^{i} d_{j} N_{j, 3}(u), u \in\left[u_{i}, u_{i+1}\right] \subset\left[u_{3}, u_{n+3}\right]
$$

where $d_{j}(j=0,1, \cdots, n+2)$ and u are control point and knots vector of B-spline, respectively. In the paper, knots vector $U$ are calculated using the algorithm of Riesenfeid (1975).

$$
U=\left[0,0,0,0, \frac{l_{1}+l_{2}}{L}, \frac{l_{1}+l_{2}+l_{3}}{L}, \cdots, \frac{\sum_{i=1}^{n-2} l_{j}}{L}, 1,1,1,1\right]
$$

$\mathrm{U}$ is calculated as Eq. (3).

$$
\left\{\begin{array}{l}
l_{i}=\left|P_{i}-P_{i-1}\right|, L=\sum_{i=1}^{n} l_{i}, i=1,2, \cdots, n \\
u_{0}=u_{1}=u_{2}=u_{3}=0 \\
\sum_{i=1}^{n-2} l_{j} \\
u_{i}=\frac{i=4,5, \cdots, n-1}{L}, i=u_{n}=u_{n}=u_{n}=1
\end{array}\right.
$$

$$
\text { where } U \text { is rewritten as } U=\left[u_{0}, u_{1}, \cdots u_{i}, u_{n+3}\right] \text {. }
$$

Characteristic-points and the corresponding knots are put it into a mathematical formula (1), which can be expressed in matrix form:

$$
\left[\begin{array}{ccccc}
1 & & & & \\
a_{2} & b_{2} & c_{2} & & \\
& \ddots & \ddots & \ddots & \\
& & a_{n} & b_{n} & c_{n} \\
& & & & 1
\end{array}\right]\left[\begin{array}{l}
d_{1} \\
d_{2} \\
\vdots \\
d_{n} \\
d_{n+1}
\end{array}\right]=\left[\begin{array}{l}
e_{1} \\
e_{2} \\
\vdots \\
e_{n} \\
e_{n+1}
\end{array}\right]
$$

In matrix, $a_{i}, b_{i}, c_{i}$ and $e_{i}$ are calculated as follows

$$
\begin{aligned}
& a_{i}=\frac{\left(\Delta_{i+2}\right)^{2}}{\Delta_{i}+\Delta_{i+1}+\Delta_{i+2}} \\
& b_{i}=\frac{\Delta_{i+2}\left(\Delta_{i}+\Delta_{i+1}\right)}{\Delta_{i}+\Delta_{i+1}+\Delta_{i+2}}+\frac{\Delta_{i+1}\left(\Delta_{i+2}+\Delta_{i+3}\right)}{\Delta_{i+1}+\Delta_{i+2}+\Delta_{i+3}} \\
& c_{i}=\frac{\left(\Delta_{i+1}\right)^{2}}{\Delta_{i}+\Delta_{i+1}+\Delta_{i+2}}, i=1,2, \cdots, n \\
& e_{i}=\left(\Delta_{i+1}+\Delta_{i+2}\right) p_{i-1}, i=2,3, \cdots, n \\
& e_{1}=p_{0}+\frac{\Delta_{3}}{3} \dot{p}_{0}, e_{n+1}=p_{n}-\frac{\Delta_{n+2}}{3} \dot{p}_{n+1}
\end{aligned}
$$

Boundary condition of B-spline is represented as Eq. (5).

$$
\left\{\begin{array}{l}
d_{0}=p_{0} \\
d_{n+2}=p_{n} \\
\dot{p}_{0}=\dot{p}\left(u_{3}\right)=\frac{3}{\Delta_{3}}\left(d_{1}-d_{0}\right) \\
\dot{p}_{n}=\dot{p}\left(u_{n+3}\right)=\frac{3}{\Delta_{n+2}}\left(d_{n+2}-d_{n+1}\right)
\end{array}\right.
$$

where $\Delta_{i}$ is forward difference of $\mathrm{u}$ and $\Delta_{i}=u_{i+1}-u_{i}$. There are $n+3$ points obtained by resolving equation group (4), and cubic B-spline can be obtained through points $p_{i}(i=0,1, \cdots, n)$.

\section{Surface Reconstruction Based on Bi-Cubic B-Spline}

B-spline surface is of great importance in computer-aided design. Through the analysis of characteristics of the parts surface, the point clouds as characteristic points of B-spline surface are obtained using CMM, which must be in rectangular topology array. The reconstruction of the surface is that control points calculated based on characteristic points of the surface, and the B-Spline surface equation is calculated. Characteristic points of the surface is represented 
as $P_{i, j}(i=0,1, \cdots, m ; j=0,1, \cdots, n)$ and surface equation based the bi-cubic B-spline can be represented as follows

$$
\begin{aligned}
& P_{i, j}(u, v)=\sum_{i=0}^{m+2} \sum_{j=0}^{n+2} d_{i, j} N_{i, 3}(u) N_{j}(v) \\
& \Rightarrow P_{i, j}(u, v)=\sum_{i=0}^{m+2}\left(\sum_{j=0}^{n+2} d_{i, j} N_{j}(v)\right) N_{i, 3}(u)
\end{aligned}
$$

$$
\begin{aligned}
& \text { Furthermore, } P_{i, j}(u, v) \text { is rewritten as follows } \\
& \qquad P_{i, j}(u, v)=\sum_{i=0}^{m+2} C_{i}(v) N_{i, k}(u) \\
& C_{i}(v)=\sum_{j=0}^{n+2} d_{i, j} N_{j, 3}(v)
\end{aligned}
$$

Reverse calculation method of surface control points based on bi-cubic B-spline has two procedures.

1) Fixed $V$ value of these curves, and sectional curves are calculated using B-spline curve interpolation algorithm in $U$, and control points $C_{i}(v), i=0,1, \cdots, m+2$ are calculated;

2) Fixed $U$ value of these curves, and sectional curves are calculated using B-spline curve interpolation algorithm in $V$, and control points is represented as

$$
d_{i, j}, i=0,1, \cdots, m+2 ; j=0,1, \cdots, n+2 \text { are calculated. }
$$

Bi-cubic B-spline surface can be confirmed when control points are calculated; the mathematic model of form error is described by the bi-cubic B-spline surface.

\section{3d Cad Modeling Method of Form Error Based on Matlab and $\mathrm{Pro} / \mathrm{E}$}

This paper proposes a modeling method of the geometry form error based on bi-cubic B-spline, and rigorous mathematical model of form error can be set up. Because of representation of three-dimensional surface based on point matrix in Matlab, the 3D solid model of form error using for assembly simulation calculation cannot be established. Data transmission method between Matlab and CAD system is proposed and it solves the problems existing in the data transmission between mathematical model point matrix and CAD system.

Firstly, coordinate measuring machine (CMM) was used to measure geometrical surface of parts to produce point clouds. Mathematical model of form error is established using the algorithm based on bi-cubic B-spline, and the reconstruction of the surface is completed. Coordinates of points in u directional curves and $\mathrm{v}$ directional curves are stored in IBL document consistent with the Pro/E format. IBL document is modified, and then imported into Pro/E. Finally, the 3D solid model of form error in Pro/E is generated. In order to satisfy the requirements of Pro/E data format, “Open Arclength” and "Begin Section” are inserted beginning of the file. "Begin Curve" is inserted beginning of the each curve, and the three-dimensional coordinate of the each point is separated by space. IBL file format rewritten as follows:

Open (or Closed) Arclength (or Pointlength)

( Open Curve: Open, or Closed; different amounts in curve: Arclength, or Pointlength)

Begin Section ! 1

Section 1

Begin Curve ! 1

the first curves contained in Section 1

$1 \mathrm{x} 1 \mathrm{y} 1 \mathrm{z} 1$ curves contained in Section 1

the first point coordinate in the first

2 x2 y2 z2

Begin Curve ! 2

the second curves contained in Section 1

Begin Section ! 2

Section 2

Begin Curve ! 1 Section 2

the first curves contained in

\section{CASE}

To verifies the efficiency of the proposed method, four plate parts are machined on X5050 numerical control machine tool, whose size is $115 \mathrm{~cm} \times 60 \mathrm{~m} \times 15 \mathrm{~m}$, material is 45 steel, using processing techniques first rough- milling and then finish-milling. After machined, machined surfaces are measured using PMM12106G CMM, whose uncertainty measurement is $(0.6 \pm 1 / 600)$ um, and it is much lower than the machined error. The surfaces are measured at $5 \mathrm{~mm}$ sampling spaces, and there are $23 \times 11$ measurement points obtained from CMM on each plate parts. Form error models based on bi-cubic B-spline surface reconstruction are showed in Figures 1.

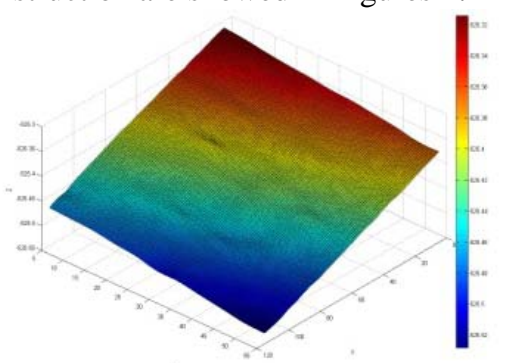

(a) 


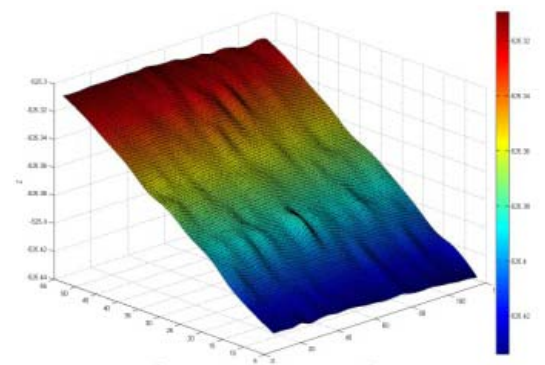

(b)

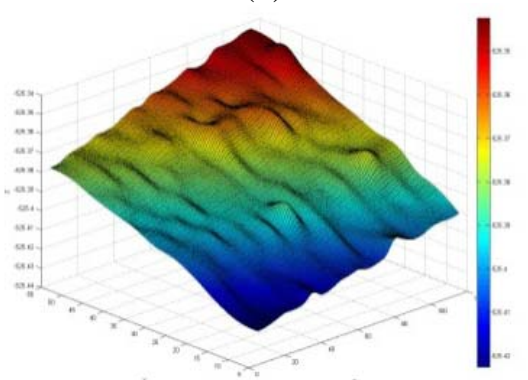

(c)

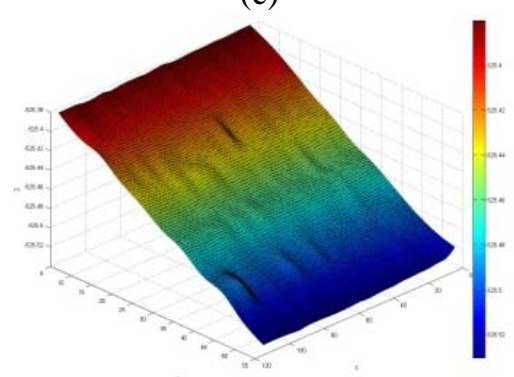

(d)

FIGURE I. FORM ERROR MODEL BASED ON BI-CUBIC B-SPLINE SURFACE RECONSTRUCTION.

The 3D form errors of a common flat and cylindrical surface are established. For the purpose of showing the surfaces more clearly, the surface of form error is enlarged by 4 times in Figure 2(a), and 100 times in Figures 2 (b) and (c).

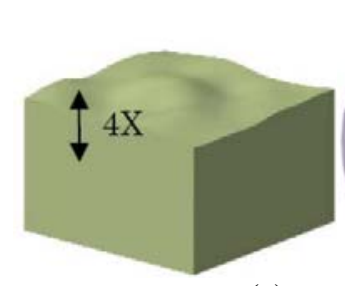

(a)

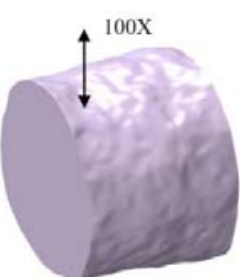

(b)

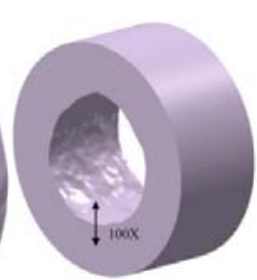

(c)
FIGURE II. CAD MODEL WITH FORM ERROR

\section{SUMMARY}

The influencing mechanism of the machined error on the assembly performance is mainly reflected in contact state and assembly accuracy between mating surfaces, and different contact state would lead to different assembly error and stress distributions. The form error and assembly force result in non-uniform contact state between mating surfaces, and thus non-uniform stress in components, which will degrade the assembly accuracy. At present, form errors of mating surfaces have significant impact on assembly accuracy, which gradually attracts the academic attentions, but assembly simulation and analysis of precision mechanical system takes into Account Plane Form Error but neglect the importance of form error and 3D distribution of form error. In order to make quantitative analysis on the surface geometric error and analyze form error and the Three dimensional distribution effective rules of the geometrical error on assembly accuracy, this paper proposes a modeling method of machined surface form error based on bi-cubic B-spline surface reconstruction, study on Data transmission method between Matlab and Pro/E, CAD solid model of parts with form error is established, it can provide theoretical foundation for the further research of effective rules of the geometrical error on assembly accuracy.

\section{ACKNOWLEDGEMENT}

This project is supported by the National Natural Science Foundation of China (Grant No. 51375054) and the Electrical-Optical Micro-Nano Manufacturing Program of Beijing Emphasis Course.

\section{REFERENCES}

[1] Turner JU and Woany MJ. Tolerances in Computer-Aided Geometric Design. The Visual Computer 1987;3:214-216.

[2] Goto M and lizuka K. A method for evaluating form errors of cylindrical parts. Japan Soc Prec Eng 1975;41(5):.

[3] Henke RP and Summerhays KD et al. Methods for evaluation of systematic geometric deviations in machined parts and their relationship to process variables. Precision Eng 1999;23:273-292.

[4] Capello E, Semeraro A. Harmonic fitting approach for plane geometry measurements. Int J Adv Manufacturing Technol 2000;16:250-258.

[5] Samper S. and Formosa F. Form Defects Tolerancing byNatural Modes Analysis. Transactions of the ASME 2007; 7:44-51.

[6] Huang WZ and Ceglarek D. Mode-based Decomposition of Part Form Error by Discrete-Cosine-Transform with Implementation to Assembly and Stamping System with Compliant Parts. Annals of the CIRP 2002;51 (1):21-26.

[7] R.S.Srinivasan,K.L.Wood. Geometric tolerancing in mechanical design using fractal-based parameters. Journal of Mechanical Design, 1995, 117: 203-206.

[8] Grandjean J, Ledoux Y, Samper S, et al. Form Errors Impact in a Rotating Plane Surface Assembly[J]. Procedia CIRP, 2013, 10: 178-185.) 